\title{
Research on Collaborative Innovation Process of Industry-university-research Institute Based on Knowledge Transfer
}

\author{
Xue $\mathrm{Li}^{1,}$, , Wenman Chen ${ }^{1, b}$ \\ ${ }^{1}$ School of Management, Wuhan University of Technology, Wuhan 430070, China. \\ a370637160@qq.com, b 619472911@qq.com
}

Keywords: Knowledge transfer, Industry-university-research institute collaborative innovation, SECI theoretical model.

\begin{abstract}
With the deepening of the theory and practice of industry-academy-research cooperation, the collaborative innovation of industry-university-research(IUR) has become the strategic measure to enhance the national independent innovation ability. Based on this, this paper summarizes the concept and process of collaborative innovation and knowledge transfer, and proposes that the process of knowledge transfer across different organizations can be divided into three stages: knowledge sharing, knowledge evaluation and knowledge absorption, and then builds the SECI theoretical model of knowledge transfer based on collaborative innovation process. By analyzing the characteristics of knowledge transfer in IUR collaborative innovation, this paper explores the process essence of IUR institute collaborative innovation and puts forward relevant countermeasures and suggestions to improve the knowledge transfer level and promote collaborative innovation.
\end{abstract}

\section{Introduction}

Since 1912 Schumpeter put forward the theory of innovation, scholars have been deepening the research for innovation, innovation theory continues to develop. Knowledge transfer is an important component in the process of collaborative innovation of IUR, which makes the knowledge circulate across innovation organizational boundaries ${ }^{[1]}$. This paper studies the process of collaborative innovation of industry-university-research from the perspective of knowledge transfer, constructs the SECI theoretical model of knowledge transfer under the cooperation mode of IUR, analyzes its internal operating mechanism, and puts forward corresponding countermeasures and suggestions. It has great significance to promote the rapid and accurate flow of knowledge between enterprises, universities and research institutes, to improve innovation performance of innovation subjects.

\section{Cooperative Innovation of IUR and Knowledge transfer Process}

Industry-university-research institute collaborative innovation is the three basic subjects to invest their respective advantages of resources and capabilities, with the support of the government, science and technology service intermediaries, financial institutions and other supporting organizations, collaborative knowledge and technology research and development innovation activities, its essence is cross-organizational collaborative innovation.

The Basic Process of IUR Cooperative Innovation. Based on the above-mentioned basic understanding of collaborative innovation, the three basic subjects, with their own development strategy and innovation capability as the goal, invest their own innovative resources, complement each other, in order to realize the cross-organizational of resources interaction, sharing and integration, enhance the innovative ability of the main innovation and core competitiveness. Therefore, the basic process of IUR collaborative innovation is not only the process of innovation subjects resource agglomeration, but also the process of innovation demand realization and value increment. The basic process of collaborative innovation is composed of a series of innovation activities, which include the aggregation, flow and creation of resource flows, which are integrated with the innovation chain(Fig. 1). 


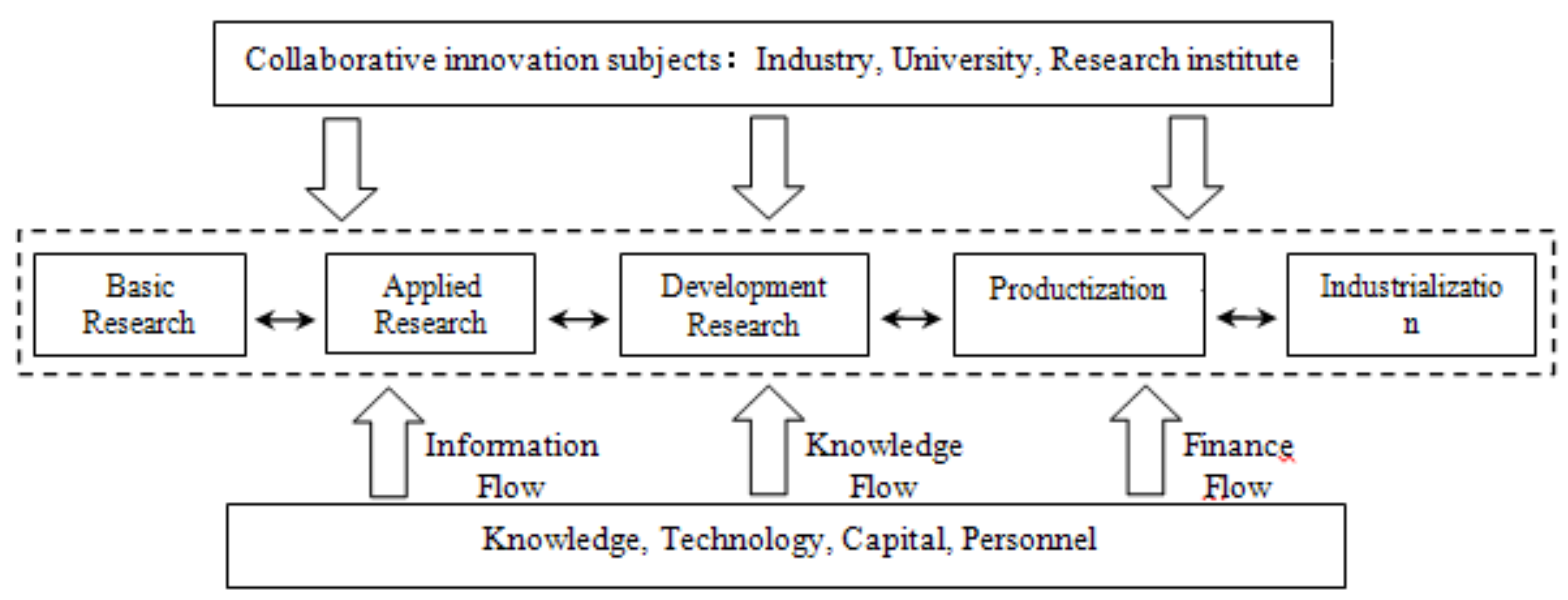

Fig. 1 The Basic Process of IUR Collaborative Innovation

There are three basic innovation subjects in the collaborative innovation system, which are university, industry, and research institute respectively ${ }^{[2]}$.Under the common support of the government, intermediary organizations and financial institutions, the three subjects are guided by the principles of complementary advantages, risk sharing, benefit sharing and win-win cooperation, based on the unique and innovative resources of the parties, which resulte in achieving the overall co-innovation effect that a single subject can not be, and achieving a strong economic impetus.

Based on this, a series of innovation activities in the process of collaborative innovation are manifested in a form of innovation chain, which includes five major innovation activities, namely basic research, applied research, development research, productization and industrialization. In every stage of innovation, there is a process of knowledge transfer among different innovation subjects. In the basic process of IUR collaborative innovation, the three main innovation subjects carry out the innovation activities in different stages of innovation chain, so that the mutual flow of knowledge, information and finance can be formed among the subjects and this cross-organizational approach can resulte in a steady stream of competitive advantages and "1+1>2" synergies.

Knowledge Transfer Process. Collaborative innovation of industry - university - research is a process of knowledge transfer, knowledge application and knowledge innovation ${ }^{[3]}$. In this paper, the whole process of knowledge transfer is divided into two stages: the first stage, the front-end of knowledge transfer, primitive accumulation of knowledge that is the source of knowledge, the basic carrier is the basic scientific knowledge. It is toward the most basic and most complex two directions to expand, and constantly breeds a new major breakthrough, accelerating cross-integration, savings energy for the new knowledge transfer. The second stage is the back-end of knowledge transfer, that is, the knowledge of one subject has been successfully absorbed by another, this stage contains the main part of knowledge transfer(Fig. 2).

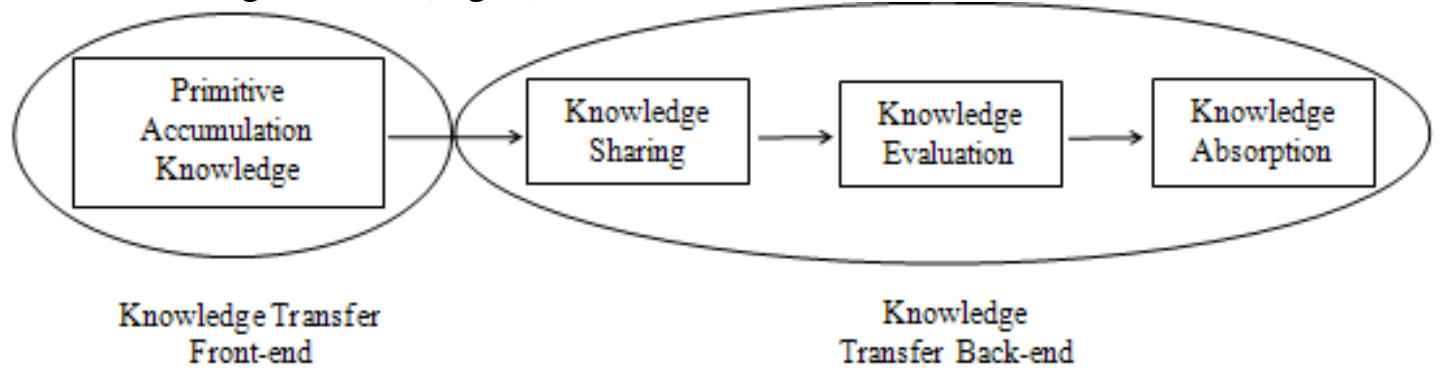

Fig. 2 A Simple Linear Model of The Basic Process of Knowledge Transfer

At the front-end of knowledge transfer, the elements included is the knowledge source, that is, the knowledge stock accumulated in the primitive. The basic approach to the primitive accumulation of knowledge is to carry out basic scientific research, which refers to the research activities of understanding natural phenomena, revealing the laws of nature ${ }^{[4]}$, acquiring new knowledge, new principles and new methods. At the back-end of knowledge transfer, the elements include three aspects, namely, knowledge sharing, knowledge evaluation and knowledge absorption. Knowledge sharing is the process of presenting knowledge to another subject by a subject with a certain 
knowledge. Knowledge evaluation is essentially the selection and reconstruction of knowledge. In the whole process of knowledge transfer, the knowledge evaluation link is also the process of communication between the knowledge exporter and the knowledge recipient. Knowledge absorption refers to the process of transforming the knowledge which is not known beforehand into various understandable knowledge and merging them into the existing knowledge base.

\section{Internal Mechanism and Suggestions of Knowledge Transfer in Collaborative Innovation}

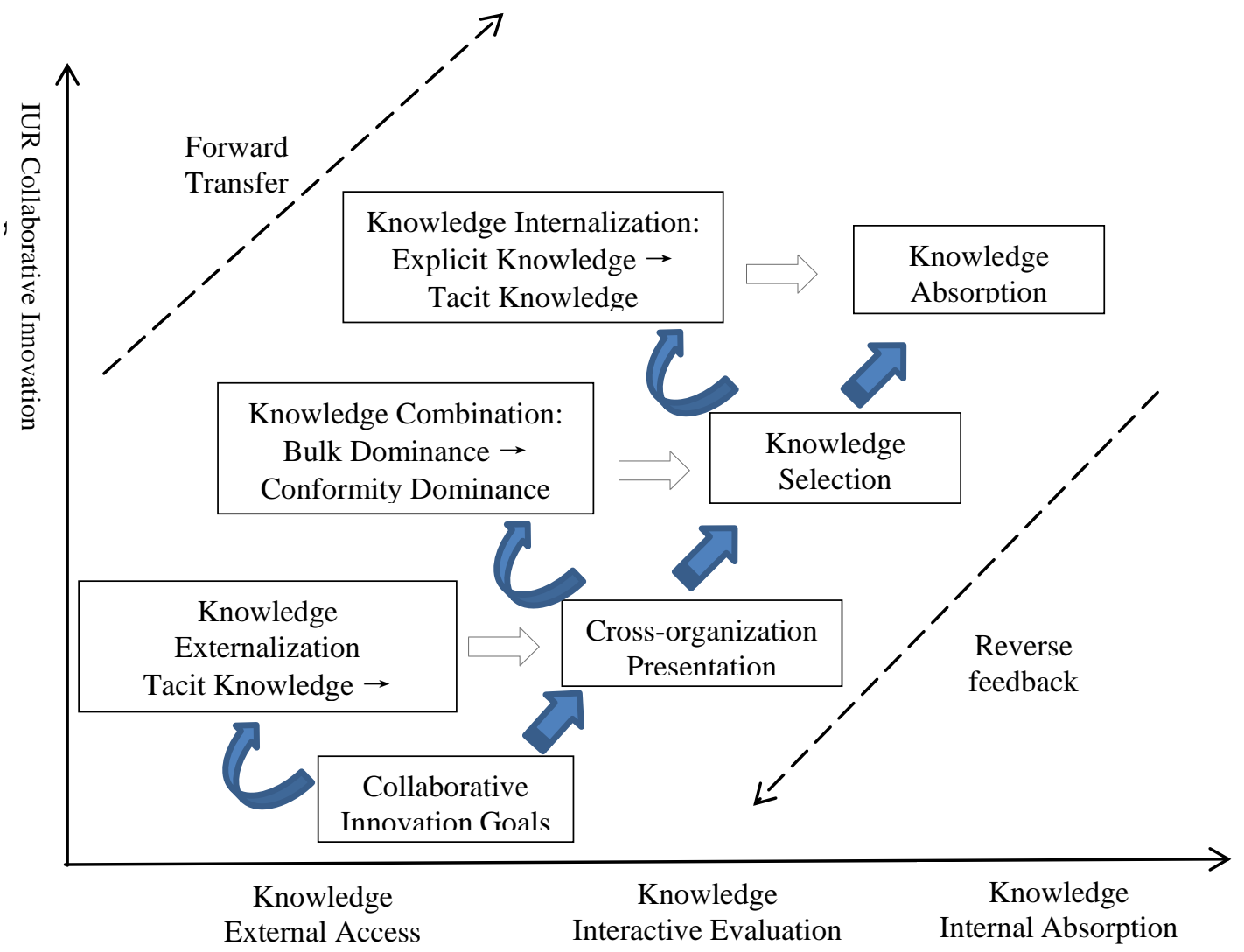

Fig. 3 SECI Theoretical Model of Knowledge Transfer in IUR Collaborative Innovation

The Internal Mechanism of Knowledge Transfer. According to the characteristics of explicit and implicit classification of knowledge and liquidity, this paper divides the knowledge transfer process in IUR collaborative innovation into three stages: knowledge acquisition, knowledge interaction evaluation and knowledge internal absorption. In the view of innovation chain, it can be seen that knowledge transfer runs through every link of the whole innovation chain. Knowledge transfer is carried out at each stage to realize the innovation output and transformation of knowledge(Fig. 3).

The externalization stage refers to the transformation of tacit knowledge, which is a process of explicit expression of tacit knowledge with explicit concepts and language. The combination stage is the key link of the knowledge transformation ${ }^{[5]}$. It refers to the process of transforming the single dominant knowledge into the explicit knowledge of the cooperative organization and realizing the knowledge increment.It is a process of combining and systematizing the explicit concept through language or number sign produced by various media. The internalization stage is the process of transforming the explicit knowledge of the cooperative organization into a tacit knowledge absorbed and understood by a single subject. It is a process of visualizing and embodying explicit knowledge, and the new explicit knowledge is absorbed and digested by the innovator, and sublimated into the tacit knowledge of the subject of knowledge transfer.

\section{Suggestions.}

(1)Knowledge potential difference as the premise 
As the knowledge potential difference leads to knowledge diffusion, and under the effect of knowledge innovation dynamic mechanism, realizing knowledge innovation, so that make the existence of knowledge potential difference absolutely. Enterprises have more knowledge of experience form than schools and research institutions, resulting in "production - research" trend knowledge transfer; On the contrary, universities and research institutes have more knowledge of theoretical form than enterprises, resulting "academic research-production" trend knowledge transfer.

(2)Organizational learning as a link

The knowledge transfer in the collaborative innovation of industry-university-research is based on the organizational learning among the innovative subjects. Some studies have shown that the interactive learning between enterprises can improve the innovation performance of enterprises in cooperative organizations ${ }^{[6]}$. Enterprises and universities, research institutes and other innovative interactive learning, access to complementary resources, technology, knowledge and ability to form a competitive advantage.

(3)Clarify the ownership of intellectual property rights and reasonable distribution of benefits for the protection

The ownership of the intellectual property rights among the innovation subjects clear and the distribution of the benefits reasonable is the foundation of forming the stable IUR innovation mechanism. In the event of disputes, the efficiency of knowledge transfer in IUR collaboration will drop, stagnate and even terminate. In order to avoid conflicts of interest distribution, a reasonable contract is needed to define the ownership of intellectual property rights and to stipulate the distribution of benefits. The contract-based trust provides the guarantee for the knowledge sharing and delivery of the innovation subjects, and effectively promotes the knowledge transfer in the collaborative innovation.

\section{Summary}

The main subjects of IUR cooperating innovation combines the core competitive advantage, and constructs the organizational structure of the unity of target and resource integration. This paper analyzes the process of industry-university-research institute collaborative innovation and the key links of knowledge transfer, constructs the SECI theoretical model of knowledge transfer from three aspects, which are knowledge externalization, combination and internalization, analyzes the basic characteristics of knowledge transfer in the process of collaborative innovation, and puts forward relevant countermeasures and suggestions, with a view to enrich and develop the theory of collaborative innovation.

\section{References}

[1] James Danowski. Identifying Collaborative Innovation Networks at The Departmental Level[J]. Procedia Social and Behavioral Sciences, 2010, 2(4): 6404-6417.

[2] Bonaccorsi Piccalnga. A Theoretical Framework for The Evaluation of University Industry Relationships [J]. R\&D management, 1994, 3(24): 120-123.

[3] Chenglei Li, Lei Ye. Study on Cooperation Model and Cooperation Process of Industry University Research Cooperation Based on Interactive View [J] Science and Technology Progress and Policy, 2011,28 (24): 30-33. (In Chinese)

[4] McKelvey M., Almb H., Riccaboni M.. Does Co-location Matter for Formal Knowledge Collaboration in Swedish Biotechnology Sector [J]. Research Policy, 2003, 32: 483-501

[5] Kaiming Wang, Junkang Wan. On the Transfer and Diffusion of Knowledge [J]. Foreign Economic and Social Management, 2000, (10): 2-7. (In Chinese) 
[6] Yubing Yang, Hanhui Hu. Network Structure and Knowledge Transfer [J] .Science and Science and Technology Management, 2008, (2): 123-127. (In Chinese) 